\title{
Gerenciamento de projetos: contribuições para a governança de TI no setor público brasileiro
}

Bruno Campelo Medeiros

Professor do Instituto Federal de Educação, Ciência e Tecnologia do Rio Grande do Norte (IFRN) Doutorando em Administração pela Universidade Federal do Rio Grande do Norte (UFRN), Rio Grande do Norte, Brasil brunocampeloufrn@ufrn.gmail.com

Miler Franco Danjour

Professor do Instituto Federal de Educação, Ciência e Tecnologia do Rio Grande do Norte (IFRN) Doutorando em Administração pela Universidade Federal do Rio Grande do Norte (UFRN), Rio Grande do Norte, Brasil

miler.danjour@ifrn.edu.br

Manoel Veras de Sousa Neto

Doutor em Administração pela Universidade de São Paulo (USP)

Professor do Programa de Pós-graduação em Administração da Universidade Federal do Rio Grande do Norte (UFRN), Rio Grande do Norte, Brasil manoel.veras@uol.com.br

\section{Editor Científico: José Edson Lara}

Organização Comitê Científico

Double Blind Review pelo SEER/OJS

Recebido em 08.06.2016

Aprovado em 14.03.2017

\footnotetext{
Este trabalho foi licenciado com uma Licença Creative Commons - Atribuição - Não Comercial 3.0 Brasil
} 


\title{
Resumo
}

A presente pesquisa tem como objetivo avaliar em que medida a adoção de práticas de gerenciamento de projetos influenciam no aumento da governança de Tecnologia da Informação (TI) das instituições públicas. Para a realização desta pesquisa, considerou-se a ausência de estudos que possam comprovar a relação entre esses aspectos no contexto do setor público, bem como o crescimento de pesquisas relacionadas ao tema governança e elementos das práticas de gerenciamento de projetos. Para isto, foram analisadas 372 instituições públicas incluídas na lista de instituições avaliadas pelo Tribunal de Contas da União (TCU) acerca das práticas de governança de TI utilizadas. A pesquisa adotou uma abordagem quantitativa e utilizou as técnicas de correlação e regressão linear múltipla. Os resultados demonstram que as questões relacionadas às práticas de gerenciamento de projetos adotadas por estas instituições contribuem para a melhoria da governança de Tl, sendo o gerenciamento de portfólio uma das práticas de maior influência.

Palavras-chave: Gerenciamento de Projetos; Práticas de Gerenciamento de Projetos; Governança de TI; Instituições públicas.

\section{Project management: contributions for IT governance in brazilian public sector}

\begin{abstract}
This research aims to assess to what extent the adoption of project management practices influence on increasing the Information Technology (IT) governance of public institutions. To carry out this research, it's considered the absence of studies that can show the relationship between these aspects in the context of the public sector, as well as the growth of research related to the governance theme and elements of the project management practices. For this, 372 were analyzed public institutions included in the list of institutions assessed by the Tribunal de Contas da União (TCU) about IT governance practices used. The survey adopted a quantitative approach, through the techniques of correlation and multiple linear regression. The results show that the related issues and project management practices adopted by these institutions contribute to the improvement of IT governance, and portfolio management one of the most influential practices.
\end{abstract}

Keywords: Project Management; Project Management Practices; IT Governance. Public Institutions. 


\section{Gestión de proyectos: contribuciones a la gobernanza de Tl em el sector público brasileño}

\section{Resúmen}

Esta investigación pretende evaluar en qué medida la adopción de la influencia de las prácticas de la gerencia de proyecto en el aumento de la gobernanza de tecnologia de la información (TI) de las instituciones públicas. Para llevar a cabo esta investigación, se consideró la falta de estudios que puede mostrar la relación entre estos aspectos en el contexto del sector público, así como el crecimiento de la investigación relacionadas con el tema gobernanza y los elementos de las prácticas de gestión de proyectos. Para ello, 372 fueron analizados las instituciones públicas incluidas en la lista de instituciones evaluadas por el Tribunal de Contas da União (TCU) respecto las prácticas de gobernanza de TI utilizadas. La encuesta adopta un enfoque cuantitativo, a través de las técnicas de correlación y regresión lineal múltiple. Los resultados demuestran que los temas relacionados con prácticas de gestión de proyecto adoptadas por estas instituciones contribuyen a la mejora de la gobernanza y la gestión de una cartera de gran influencia.

Palavras clave: Gestión de proyectos; Prácticas de la gerencia de proyectos; Gobernanza de TI; Instituciones públicas.

\section{Introdução}

Em um contexto recente, as organizações que compõem o setor público vêm sofrendo algumas mudanças, sobretudo em suas formas estruturais. As práticas da nova gestão pública, focadas em resultados, se tornam mais relevantes neste contexto. Tal abordagem contempla a adoção, no setor público, de princípios gerenciais característicos do setor privado (Diefenbach, 2007; Ashraf \& Uddin, 2015), com o objetivo de melhorar o desempenho das organizações públicas e incluindo a estratégia como meio para o alcance dos resultados (Pablo, Reay, Dewald, \& Casebeer, 2007).

As práticas de gerenciamento de projetos vêm contribuindo nesse cenário organizacional como forma de aprimorar o setor público e aplicar esse novo modelo de gestão pública, por meio de processos de planejamento, execução de controle das políticas públicas, gerando benefícios relacionados ao controle de prazos e custos, bem como a otimização de recursos organizacionais.

Embora não seja algo recente (Meredith \& Mantel Jr., 2000), as práticas de gerenciamento de projetos tiveram a sua popularização entre os anos de 1980 e 1990, com o uso intensivo de técnicas e processos de gerenciamento de projetos, 
em face das mudanças nas estruturas organizacionais que aconteceram nesse período, estabelecendo novas formas de organização (Tsaturyan \& Muller, 2015), principalmente no que se refere à implantação de sistemas e desenvolvimento da tecnologia.

Investimentos elevados em Tecnologia da Informação (TI) no setor público têm sido realizados. Com os projetos de $\mathrm{TI}$ demandando mais recursos, há a necessidade de utilizar estruturas de controle no gerenciamento desses projetos, de forma que eles garantam que os objetivos sejam atendidos, obtendo um desempenho melhor da TI (Muller \& Martinsuo, 2015). Nesse contexto, a preocupação em termos de desempenho da TI está associada a mecanismos de governança que promovam um maior controle e alinhamento estratégico entre a área de TI e a organização, traduzindo, assim, em um melhor desempenho. Estudos recentes mostram que a eficácia da governança de TI contribui para a melhoria do desempenho organizacional (Heindrickson \& Santos Jr., 2014; Mendonça, Guerra, Sousa Neto, \& Araújo, 2013; Mohamed \& Singh, 2011). Medir a governança de TI ajuda a entender como está o desempenho da TI em termos de agregação de valor organizacional.

Assim, diversas práticas com foco em governança são utilizadas para medir o desempenho da $\mathrm{TI}$ e sua contribuição para o negócio. Baseado no modelo COBIT (Control Objectives for Information and related Technology), um guia de boas práticas que reúne os aspectos relacionados aos objetivos de controle do negócio que precisam ser atendidos pela área de TI das organizações, o Tribunal de Contas da União (TCU) estabelece uma avaliação bienal acerca do nível de governança dos processos de TI, realizada nos diversos órgãos da Administração Pública Brasileira, onde são avaliados diversos aspectos relativos às práticas de governança e de gestão de $\mathrm{TI}$, e, com base na nota atribuída à organização avaliada, tem-se o nível de maturidade da governança dessas instituições.

Tratar de governança de TI é uma preocupação da administração pública, no sentido de garantir que os recursos de TI sejam bem aplicados e aderentes às necessidades institucionais, com objetivos claros e alinhados. Diante deste contexto, o presente estudo tem como objetivo avaliar em que medida a adoção de práticas de gerenciamento de projetos influencia no aumento da governança de TI das instituições públicas. Como justificativa para o estudo, destaca-se que as questões 
relacionadas às práticas de gerenciamento de projetos são pouco esclarecidas no contexto das organizações públicas, sobretudo na área de TI.

A literatura sobre o tema proposto não apresenta um volume consistente de pesquisas empíricas que testem e validem esses conceitos e técnicas (Rosacker \& Rosacker, 2010). Em se tratando de gerenciamento de projetos de TI, pesquisas recentes adotaram vertentes distintas, relacionadas às normas de relacionamento (Muller \& Martinsuo, 2015), às habilidades gerenciais (Keil, Lee \& Deng, 2013), à aplicação de princípios de governança ao campo de gerenciamento de projetos (Bernroider \& Ivanov, 2011; Marnewick \& Labuschagne, 2011) e aplicação de gerenciamento de projetos de TI no setor público, tratando esse tema de forma conceitual (Rosacker \& Rosacker, 2010).

Ao mesmo tempo, outras pesquisas, dentro de um contexto mais amplo, apresentam elementos da governança de projetos, tratando de um conceito emergente (Bekker, 2015; Mosavi, 2014; Bernardo, 2014; Too \& Weaver, 2014; Aubry, Richer, \& Lavoie-Tremblay, 2014) e outras que recomendam mais estudos sobre o tema (Tsaturyan \& Muller, 2015). No entanto, não se têm estudos que tratam da relação entre as práticas de gerenciamento de projetos e suas contribuições para a melhoria da governança de TI, especialmente, nas organizações públicas.

Para alcançar o objetivo proposto, a pesquisa foi estruturada nas seguintes partes: na estrutura teórica, foram discutidos os principais conceitos sobre governança de $\mathrm{Tl}$, gerenciamento de projetos e governança em projetos, além das hipóteses da pesquisa; em sequência, apresenta-se com a descrição dos métodos utilizados; a penúltima parte descreve os resultados obtidos com o estudo e sua discussão; por fim, tem-se a conclusão do estudo, com uma síntese dos principais resultados, limitações e sugestões para estudos futuros.

\section{Governança de Ti Gerenciamento de Projetos e Governança em Projetos}

A governança de TI pode ter um importante papel na definição da estrutura de $\mathrm{TI}$ adotada nas organizações. Além de oferecer maior transparência da administração nos negócios para os stakeholders, ela define um padrão de ações e tomada de decisão para a área de TI e promove o alinhamento da área de TI em 
relação aos negócios, por meio de um gerenciamento de serviços eficaz e controle mais adequado dos processos.

O conceito de governança de $\mathrm{Tl}$ tem sua origem no conceito de governança corporativa, que é um conceito mais amplo, no sentido organizacional. Com o processo de profissionalização das organizações, originado no início do século $\mathrm{XX}$, houve a separação da propriedade do controle administrativo, ou seja, a substituição dos proprietários do negócio por executivos contratados por estes proprietários para administrar o negócio, gerando conflitos de agência entre os agentes principais (proprietários) e os agentes executores (executivos). A governança corporativa surgiu, então, como um instrumento de controle para monitorar as ações desses executivos e proteger os proprietários do negócio, por intermédio da definição de papéis e responsabilidades atribuídas aos diversos stakeholders da organização (Rossetti \& Andrade, 2011).

Em um contexto mais recente, esses processos de controle instituídos pela governança corporativa se tornaram maiores com as mudanças no cenário mercadológico, sobretudo com a criação dos mecanismos de compliance, que procuram regular melhor o mercado, exigindo das empresas maior transparência e acesso à informação aos acionistas.

A governança de $\mathrm{Tl}$ surge como um processo de controle do negócio em relação à área de $\mathrm{TI}$, não apenas pela necessidade de geração desta informação de maneira mais eficaz, em meio à constituição destes marcos regulatórios, como também na necessidade de obter uma gestão dos recursos de TI pela organização (Prasad, Heales, \& Green, 2010). Cada vez mais a alta administração está exigindo que a tecnologia da informação comprove o retorno sobre o investimento em seus projetos e que consiga agregar maios valor para o negócio da empresa (Prestes \& Brodbeck, 2011; Ho, Wu, \& Xu, 2010).

Em se tratando do gerenciamento de projetos, trata-se de uma prática que não é recente. Há bastante tempo, atividades empresariais eram desenvolvidas em forma de projetos e administradas com técnicas e metodologias de gerenciamento, por meio de planejamento, controle, orçamento e cronograma de projetos. O início do gerenciamento de projetos por meio de projetos complexos se deu a partir da década de 1950, nas áreas militar e espacial, que exigiam uma maior estrutura de planejamento, organização e controle de atividades envolvidas. O projeto espacial 
Apollo, da NASA, como também o projeto Polaris, da marinha norte-americana, são alguns exemplos de estudos iniciais envolvendo a área de gerenciamento de projetos (Meredith \& mantel Jr., 2000).

$\mathrm{Na}$ visão de Dinsmore (2007), o projeto é um empreendimento único, conduzido por pessoas, utilizando recursos diversos a fim de se atingir objetivos prédefinidos. Dentro dessa conjuntura, pode-se afirmar que todo o projeto se caracteriza por ser temporário (início e fim definidos), exclusivo (destinado ao cumprimento de um ou mais objetivos específicos) e progressivo (detalhado, na medida em que se aprofunda o grau de compreensão sobre ele pelos envolvidos). Sendo assim, a prática de gerenciar projetos nas organizações ajuda no planejamento, execução e controle das atividades desenvolvidas, estabelecendo prazos e otimizando recursos, a fim de se obterem resultados cada vez mais consistentes e alinhados com a estratégia e política geral do negócio.

Ter sucesso em projetos depende de uma série de fatores que precisam estar vinculados ao desempenho organizacional (Rolstadas, Tommelein, Schiefloe, \& Ballard, 2014). Neste sentido, as práticas de gerenciamento de projetos são importantes para viabilizar o alinhamento estratégico organizacional e melhor controle desses projetos. Na conjuntura atual, com a necessidade de um maior alinhamento estratégico e controle da gestão, o desenvolvimento de estudos recentes que associam as práticas de gerenciamento de projetos aos conceitos de governança corporativa recebeu maior atenção. A Tabela 1 apresenta um resumo desses estudos:

\section{Tabela 1}

Pesquisas envolvendo gerenciamento de projetos e governança

\begin{tabular}{|c|c|c|c|}
\hline Autores & Abordagem & Foco do estudo & Amostra \\
\hline $\begin{array}{l}\text { der e } \\
(2011)\end{array}$ & Quantitativa & $\begin{array}{l}\text { Verificar a validade e usabilidade da } \\
\text { estrutura do modelo Cobit na área de } \\
\text { gerenciamento de projetos }\end{array}$ & $\begin{array}{l}578 \text { profissionais de } \mathrm{TI}, \\
\text { gerenciamento de } \\
\text { projetos e auditores de } \\
\text { sistemas }\end{array}$ \\
\hline $\begin{array}{l}\text { Marnewicl } \\
\text { Labuscha } \\
\text { (2011) }\end{array}$ & Qua & $\begin{array}{l}\text { overnança que } \\
\text { de } \mathrm{TI}\end{array}$ & $\begin{array}{l}16 \text { gestores de TI e } \\
\text { gestores de programas } \\
\text { de empresas da África do } \\
\text { Sul }\end{array}$ \\
\hline $\begin{array}{l}\text { Aubry, Richer } \\
\text { e Lavoie- } \\
\text { Tremblay } \\
\text { (2014) }\end{array}$ & Quali & $\begin{array}{l}\text { izações } \\
\text { S Os }\end{array}$ & $\begin{array}{l}45 \text { profissionais membros } \\
\text { do comitê executivo e do } \\
\text { escritório de projetos }\end{array}$ \\
\hline $\begin{array}{l}\text { Bernardo } \\
(2014)\end{array}$ & $\begin{array}{l}\text { Métodos } \\
\text { mistos }\end{array}$ & $\begin{array}{l}\text { Analisar a percepção sobre a relevância } \\
\text { dos indicadores de desempenho em }\end{array}$ & $\begin{array}{l}33 \text { gestores de projetos, } \\
\text { patrocinadores, membros }\end{array}$ \\
\hline
\end{tabular}




\begin{tabular}{|c|c|c|c|}
\hline & & $\begin{array}{l}\text { projetos no contexto da governança de } \\
\text { portfolios, programas e projetos }\end{array}$ & $\begin{array}{l}\text { de equipe e fornecedores } \\
\text { de alto nível de } 6 \\
\text { empresas }\end{array}$ \\
\hline $\begin{array}{l}\text { Biesenthal e } \\
\text { Wilden (2014) }\end{array}$ & Teórica & $\begin{array}{l}\text { Identificar os conceitos predominantes e } \\
\text { temas subjacentes acerca da governança } \\
\text { de projetos }\end{array}$ & - \\
\hline $\begin{array}{l}\text { Guo et al. } \\
(2014)\end{array}$ & Qualitativa & $\begin{array}{l}\text { Investigar como diferentes estruturas de } \\
\text { governança de projetos afetam a gestão } \\
\text { de riscos }\end{array}$ & $\begin{array}{l}42 \text { representantes de } \\
\text { stakeholders de } 2 \\
\text { projetos de infraestrutura } \\
\text { na Nova Zelândia }\end{array}$ \\
\hline $\begin{array}{l}\text { Hjelmbrekke, } \\
\text { Laedre e } \\
\text { Lohne (2014) }\end{array}$ & Qualitativa & $\begin{array}{l}\text { Descobrir o que os patrocinadores do } \\
\text { projeto podem fazer para garantir a } \\
\text { criação e valor dos seus projetos }\end{array}$ & $\begin{array}{l}12 \text { patrocinadores de } \\
\text { projetos, sendo } 6 \\
\text { públicos e } 6 \text { privados }\end{array}$ \\
\hline Mosavi (2014) & Qualitativa & $\begin{array}{l}\text { Explorar os papeis dos conselhos } \\
\text { diretivos na governança de portfolio de } \\
\text { projetos }\end{array}$ & $\begin{array}{l}32 \text { gestores de } 3 \\
\text { empresas } \\
\text { dinamarquesas }\end{array}$ \\
\hline $\begin{array}{l}\text { Muller e } \\
\text { Lecoeuvre } \\
(2014)\end{array}$ & Quantitativa & $\begin{array}{l}\text { Desenvolver categorias para a } \\
\text { operacionalização da governança de } \\
\text { projetos }\end{array}$ & $\begin{array}{l}478 \text { profissionais de } \\
\text { gerenciamento de } \\
\text { projetos }\end{array}$ \\
\hline Pinto (2014) & Qualitativa & $\begin{array}{l}\text { Explorar as formas pelas quais a } \\
\text { normalização do desvio impacta na } \\
\text { entrega dos projetos em grandes } \\
\text { organizações }\end{array}$ & $\begin{array}{l}21 \text { gestores de projetos } \\
\text { de } 3 \text { empresas }\end{array}$ \\
\hline $\begin{array}{l}\text { Too e Weaver } \\
(2014)\end{array}$ & Teórica & $\begin{array}{l}\text { Examinar os conceitos existentes sobre } \\
\text { governança de projetos e gerenciamento } \\
\text { de projetos }\end{array}$ & - \\
\hline Bekker (2015) & Teórica & $\begin{array}{l}\text { Investigar o conceito de governança de } \\
\text { projetos }\end{array}$ & - \\
\hline Chang (2015) & Teórica & $\begin{array}{l}\text { Demonstrar que o conceito de } \\
\text { capacidade de assumir riscos pode ser } \\
\text { utilizado como uma nova dimensão para } \\
\text { a análise do conceito de governança de } \\
\text { projetos }\end{array}$ & - \\
\hline $\begin{array}{l}\text { Joslin e Muller } \\
\text { (2015) }\end{array}$ & Quantitativa & $\begin{array}{l}\text { Analisar a relação entre o uso de } \\
\text { metodologias de gerenciamento de } \\
\text { projetos, o sucesso do projeto e o } \\
\text { impacto da governança de projetos nesta } \\
\text { relação }\end{array}$ & $\begin{array}{l}254 \text { profissionais de } \\
\text { gerenciamento de } \\
\text { projetos }\end{array}$ \\
\hline $\begin{array}{l}\text { Muller e } \\
\text { Martinsuo } \\
(2015)\end{array}$ & Quantitativa & $\begin{array}{l}\text { Analisar o impacto das normas de } \\
\text { relacionamento no sucesso dos projetos } \\
\text { em diferentes contextos de governança } \\
\text { de projetos }\end{array}$ & 200 gerentes de projetos \\
\hline
\end{tabular}

Fonte: Dados da pesquisa, 2016.

Pela Tabela 1, percebe-se que os estudos desenvolvidos, em sua maioria, procuram associar as práticas de gerenciamento de projetos a um conceito de governança de projetos, identificando e avaliando elementos da governança no gerenciamento de projetos. A governança de projetos envolve a estrutura pela qual os objetivos dos projetos são definidos, bem como os meios para atingir estes objetivos e para monitorar o desempenho dos projetos, sendo conduzidos pelo fornecedor e cliente (Turner, 2006; Marnewick \& Labuschagne, 2011; Muller \& Martinsuo, 2015). 
Uma das práticas de gerenciamento de projetos associada à governança é o gerenciamento de portfólio de projetos. O gerenciamento de portfólio procura relacionar objetivos estratégicos com atividades recorrentes a atividades de projetos (Veras, 2014), por meio da priorização dos projetos mais importantes, operacionalizando um conjunto de ações direcionados ao perfil estratégico. Sem esta priorização, há o risco de falta de sincronização entre os projetos e os objetivos da empresa (Levine, 2005).

No entanto, estudos mais recentes explicam que o gerenciamento de portfólio não trata apenas do processo de tomada de decisão para a priorização de projetos, contendo também outros elementos importantes relacionados à governança. Mosavi (2014) explorou o papel dos comitês diretivos relacionados ao gerenciamento de portfólio em organizações dinamarquesas, onde foram verificados papeis distintos além do processo decisório, como os papeis de comunicação e negociação. $\mathrm{Na}$ organização em que o comitê diretivo exerce um papel comunicativo, as decisões são exercidas pela alta cúpula da organização e o comitê apenas informa as decisões tomadas. Já na organização cujo papel de negociação é predominante, o comitê desenvolve propostas para alocação de recursos e sugere quais projetos devem ser priorizados, e só então as decisões são tomadas por meio da discussão das propostas.

Em se tratando do setor público, na prática há estudos que mostram deficiências no gerenciamento de portfólio. Um estudo importante envolvendo o gerenciamento de portfólio, especificamente aplicado no setor público, foi desenvolvido por Stentoft, Freytag e Thoms (2015) envolvendo municípios dinamarqueses. O estudo constatou que o processo de gerenciamento de portfólio é centralizado em alguns municípios. No entanto, a maioria dos casos apresentou a falta de coordenação e clareza neste processo de gerenciamento, pelo fato de estas instituições serem focadas nas operações do dia-a-dia, não levando em consideração o planejamento dos projetos de desenvolvimento destes municípios.

Outra prática importante para promover a governança organizacional é a prática de gerenciamento de desempenho em projetos. Neste caso, estudos recentes estão sendo desenvolvidos com o intuito de esclarecer esta relação, como nas pesquisas de Bernardo (2014), Liu e Wang (2016) e Aubry, Richer e LavoieTremblay (2014). No estudo de Bernardo (2014), foi verificada a percepção de 
gerentes de projetos, patrocinadores e membros de equipe sobre a relevância de indicadores de projetos no contexto da governança de portfólios, programas e projetos. Verificou-se que os indicadores relacionados ao desempenho dos projetos, principalmente em relação a custos, foram considerados relevantes, assim como a satisfação da equipe de projetos, até mesmo pela alta direção.

Já a pesquisa de Aubry, Richer e Lavoie-Tremblay (2014) estabelece a temática de gerenciamento de desempenho para o contexto dos escritórios de gerenciamento de projetos (EGPs). Os EGPs também podem ser considerados estruturas associadas à governança, pois envolvem uma estrutura formal paralela à estrutura funcional, que tem por objetivo apoiar essa estrutura funcional em relação à concretização dos projetos organizacionais, utilizando de uma melhor governança de recursos, processos, metodologias e técnicas de gerenciamento de projetos, ou seja, sua relação com os demais departamentos é baseada em um leque de serviços de apoio aos projetos (Veras, 2014; Jalal \& Kosha, 2015).

É importante observar que vários estudos investigaram a relação entre EGPs e desempenho organizacional, vinculados à ideia de fatores de sucesso dos EGPs nas organizações (Spalek, 2013), abordando as estruturas de governança de diferentes EGPs em uma mesma organização, considerando aspectos estruturais, relacionais, de processos e alcance de objetivos de negócios (Tsaturyan \& Muller, 2015), ou ainda avaliando o desempenho organizacional com base em práticas de governança de projetos, tendo o EGP como mecanismo central de governança (Aubry et al., 2014). Nesse último estudo, considera-se que ainda existem contradições na percepção de gestores organizacionais sobre essa contribuição dos EGPs para a melhoria do desempenho organizacional.

O EGP pode ser considerado um elemento importante para promover a governança pelas diversas funções que ele pode realizar como forma de apoio ao gerenciamento de projetos, como o controle e a fiscalização, apresentação do status dos projetos e aconselhamento à alta direção (Dai \& Wells, 2004; Hobbs \& Aubry, 2007; Blasevic, Misic, \& Simac, 2014), bem como a própria gestão de portfólio (Patel, Patel, \& Patel, 2012).

Sobre a formalização de projetos, poucas pesquisas foram desenvolvidas até então, estabelecendo a relação deste aspecto com as questões de governança. Uma pesquisa de destaque neste assunto foi desenvolvida por Muller e Martinsuo 
(2015), que analisou o impacto das normas de relacionamento no sucesso dos projetos. Os autores ressaltam a importância de se realizar o gerenciamento de projetos com estruturas organizacionais claras e metodologias formais de gerenciamento, mas ressaltam que a flexibilidade e a informalidade em alguns aspectos podem influenciar positivamente no sucesso do projeto.

Após a discussão da literatura, foi realizada a construção do modelo de pesquisa, o qual se deu por meio dos indicadores estabelecidos relacionados às práticas de gerenciamento de projetos de TI medidos pelo TCU em instituições públicas, bem como o seu índice de governança. Além disso, foi verificada na literatura existente as pesquisas mais aderentes a esses indicadores levantados, gerando hipóteses da pesquisa, descritas na Figura 1:

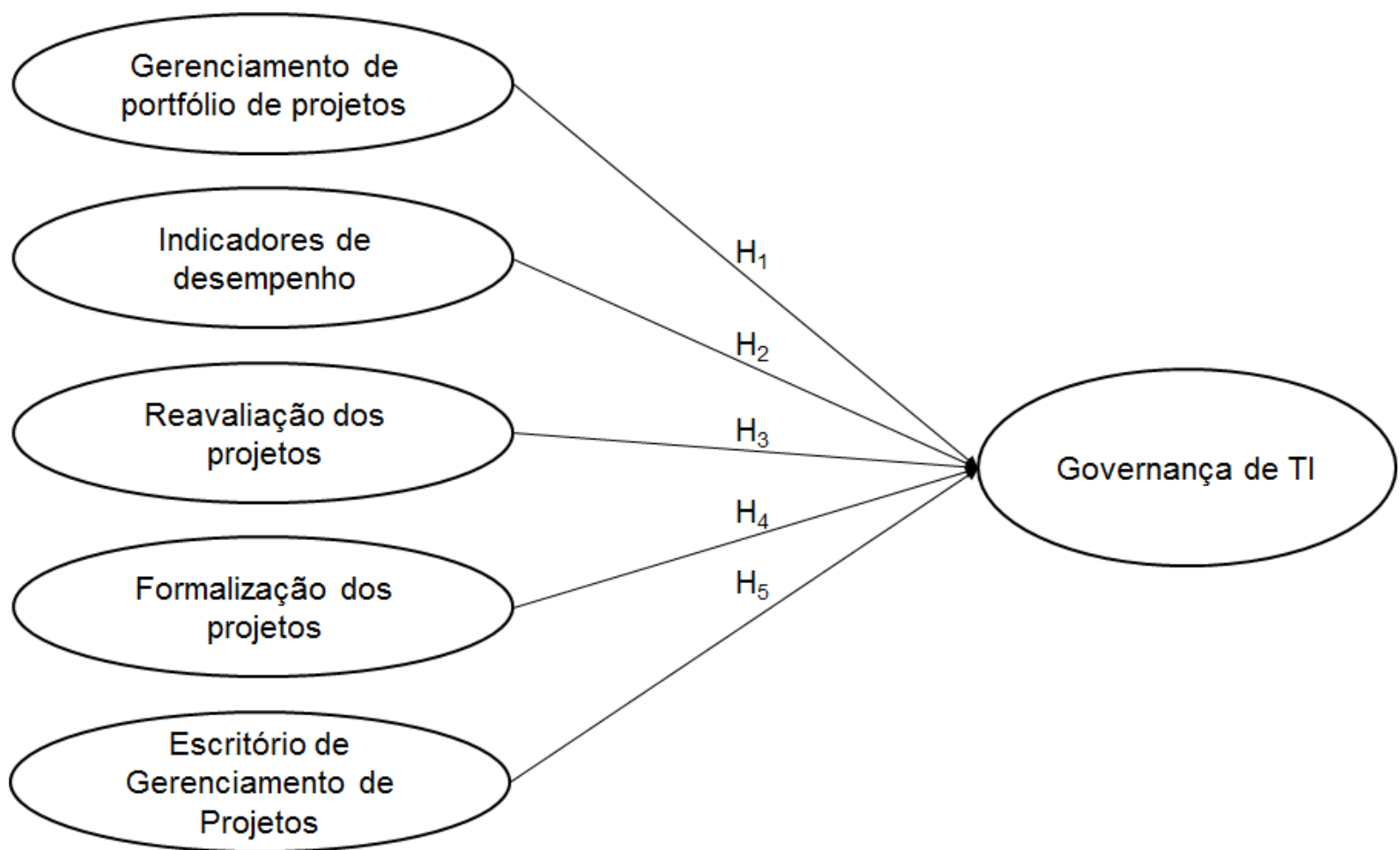

Figura 1

Modelo da pesquisa

Fonte: Dados da pesquisa, 2016.

$\mathrm{H}_{1}$ : As práticas de gerenciamento de portfólio afetam positivamente no aumento da governança de TI.

$\mathrm{H}_{2}$ : $\mathrm{O}$ uso de indicadores de desempenho em projetos afeta positivamente no aumento da governança de TI. 
$\mathrm{H}_{3}$ : O processo de reavaliação dos projetos afeta positivamente no aumento da governança de TI.

$\mathrm{H}_{4}$ : A constituição formal das práticas de gerenciamento de projetos afeta positivamente no aumento da governança de TI.

$\mathrm{H}_{5}$ : A existência de escritório de gerenciamento de projetos afeta positivamente no aumento da governança de $\mathrm{TI}$.

\section{Procedimentos Metodológicos}

O estudo utilizou uma abordagem dedutiva, que "tem o propósito de explicar o conteúdo das premissas [...]" (Marconi \& Lakatos, 2008, p.92). Essa abordagem parte de alguns postulados teóricos acerca dos aspectos da governança de TI para estabelecer conclusões sobre o assunto no contexto estudado

Para isto, a pesquisa assume a predominância do paradigma sociológico funcionalista, conforme a classificação e Burrel e Morgan (1998), com características realistas e positivistas, de explanações essencialmente racionais de assuntos sociais. Quanto aos fins, ele pode ser classificado como uma pesquisa do tipo exploratória, seguindo a estrutura conceitual de Vergara (2004), considerando que o estudo aborda questões que foram pouco exploradas na literatura, trazendo em conjunto elementos do gerenciamento de projetos e da governança de TI das instituições públicas brasileiras.

Em relação à estrutura bibliográfica, a constituição do arcabouço teórico se deu inicialmente por meio de buscas realizadas nas base de dados "Science Direct", "Periódicos Capes" e "Esmerald", tendo como palavras chave: "Governança de TI e gerenciamento de projetos", "Gerenciamento de projetos", "Governança de projetos", "IT Governance", "IT governance and Project management", "Project management and governance", e "Project governance".

Para a realização do trabalho, foi utilizada como base a fonte pública disponível no site da Secretaria de Fiscalização de Tecnologia da Informação (SEFTI) do governo federal, site vinculado ao Tribunal de Contas da União (TCU), em que se faz uma análise da governança de TI nas entidades públicas, tendo como objetivo coletar informações sobre processos de aquisição de bens e serviços, segurança da informação e gestão de recursos humanos da TI (Brasil, 2014). A 
pesquisa tomou como base os dados coletados para o período de 2014, o último levantamento divulgado pelo TCU, contendo 372 instituições, e que estão disponíveis no sítio da SEFTI (2017). A disponibilidade dos dados motivou a escolha das instituições a serem avaliadas nesta pesquisa.

O Acórdão № 3117/2014-TCU-Plenário contém os principais resultados acerca da avaliação da governança de tecnologia da informação na Administração Pública Federal, bem como recomendações gerais e oportunidades de melhoria no processo de avaliação. Realizada desde o ano de 2007, edição na qual participaram 255 organizações públicas federais, a avaliação de 2014 contou com 372 organizações da administração pública federal, tendo como principais critérios a representatividade destas organizações no orçamento da União e a autonomia da governança de TI destas instituições (Brasil, 2014).

Em relação ao tipo de análise quantitativa, as técnicas de análise utilizadas para a realização do presente trabalho foram a técnica de análise de correlação de Pearson e técnica de análise de regressão, considerando, neste estudo, a variável dependente e as demais variáveis independentes métricas. Segundo Corrar, Paulo e Dias Filho (2007), a finalidade da análise de correlação é medir o grau de relacionamento entre as variáveis. Já a análise de regressão tem o propósito de "previsão", isto é, procura prever o comportamento de uma variável dependente a partir do conhecimento de uma ou mais variáveis independentes (Hair Jr., Anderson, Tatham, \& Black, 2005).

Portanto, a especificação da equação inicial que procurará representar o modelo proposto pela pesquisa é representada da seguinte forma (Equação 1):

$$
\begin{array}{r}
\text { GovTI }=\beta_{0}+\beta_{1} \text { PortProj }+\beta_{2} \text { IndicProj }+\beta_{3} \text { ReavProj }+\beta_{4} \text { FormProj }+\beta_{5} \text { EGP }+u, \\
\text { Equação (1) },
\end{array}
$$

onde "GovTI" é uma variável dependente, que significa o índice de governança de $\mathrm{TI}$ das instituições públicas analisadas para o ano de 2014. O índice de governança de TI representa o quanto uma instituição utiliza práticas de governança de $\mathrm{TI}$, baseado em uma série de indicadores que vão desde a questão de alinhamento estratégico e controle da alta administração, até indicadores de transparência e acesso à informação pelo cidadão. O cálculo do índice de governança leva em consideração a 
nota atribuída a cada um destes indicadores, bem como a importância que cada indicador tem para a instituição analisada.

Em relação às variáveis independentes, estas possuem a seguinte classificação: "PortProj" significa o nível e adoção das práticas de portfólio de gerenciamento de projetos; "IndicProj" é uma variável que representa o uso de indicadores para o gerenciamento de projetos pelas instituições analisadas; "ReavProj" representa o quanto cada instituição reavalia os projetos de $\mathrm{Tl}$; "FormProj", envolve a constituição formal das práticas de gerenciamento de projetos; e "EGP", trata da adoção de estruturas relacionadas a escritórios de gerenciamento de projetos. Em se tratando das métricas das variáveis, devido às variáveis independentes não estarem em mesma escala métrica da variável dependente, foi estabelecido o processo de normalização das variáveis, convertendo as variáveis para uma escala percentual, eliminado, assim, o viés ocasionado pelas diferenças nas escalas das variáveis usadas na análise.

$\mathrm{O}$ processo de análise dos resultados seguiu basicamente três etapas. $\mathrm{Na}$ primeira etapa, foi feita uma análise preliminar dos dados, utilizando-se o teste de Alpha de Cronbach, para verificar a confiabilidade dos dados, em conjunto com a análise descritiva. Na segunda etapa, foi realizada a análise de correlação, estabelecendo as relações entre a variável dependente GovTI e as demais variáveis, sendo esta etapa um pré-requisito para testar as hipóteses do estudo. Na última etapa, foram realizadas as análises provenientes dos resultados obtidos com a aplicação do modelo proposto, por meio da aplicação da técnica de regressão, seguindo a estrutura de análise proposta por Hair Jr. et al. (2005) e Wooldridge (2010).

\section{Resultados}

\subsection{Análise descritiva e teste de confiabilidade do modelo}

Antes de estabelecer as correlações entre as variáveis do modelo proposto, foram realizadas a análise descritiva dos dados e a análise de confiabilidade. Ao se analisarem os resultados do índice de governança de TI das instituições, obteve-se uma média de $44,7 \%$ das práticas de governança utilizadas. Em relação às práticas 
de gerenciamento de projetos, a variável "Portfólio de Projetos" foi a que apresentou um maior nível de adoção, seguida pelas variáveis "Formalização de Projetos" e "Indicadores de Projetos", conforme mostra a Tabela 2.

\section{Tabela 2}

Análise descritiva dos dados

\begin{tabular}{l|r|r|r|r|r|r|r}
\hline \multicolumn{1}{c|}{ Variável } & $\mathbf{n}$ & Média & $\begin{array}{c}\text { Desvio- } \\
\text { padrão }\end{array}$ & Variância & Assimetria & Curtose & $\begin{array}{c}\text { Alpha de } \\
\text { Cronbach (a), se } \\
\text { retirado }\end{array}$ \\
\hline GovTI & 372 & 0,447 & 0,175 & 0,030 & 0,070 & $-0,424$ & 0,853 \\
PortProj & 372 & 0,678 & 0,231 & 0,054 & $-0,009$ & $-1,303$ & 0,851 \\
IndicProj & 372 & 0,614 & 0,212 & 0,045 & 0,308 & $-0,960$ & 0,847 \\
ReavProj & 372 & 0,585 & 0,208 & 0,043 & 0,526 & $-0,839$ & 0,840 \\
EGP & 372 & 0,574 & 0,219 & 0,048 & 0,846 & $-0,490$ & 0,845 \\
FormProj & 372 & 0,632 & 0,263 & 0,069 & 0,374 & $-1,490$ & 0,872 \\
\hline
\end{tabular}

Fonte: Dados da pesquisa, 2016.

Além da estatística descritiva apresentada na Tabela 2, foi realizado o teste de confiabilidade dos dados por meio do teste de Alfa de Cronbach. O teste de confiabilidade procurar medir o grau em que um conjunto de variáveis é consistente com o que se pretende medir, ou seja, procura testar a eficiência do modelo proposto (Hair Jr. et al., 2005). Ao realizar a análise conjunta das variáveis, o valor de Alfa de Cronbach obtido foi de 0,873 , sendo superior ao valor recomendado pela literatura, que é de 0,7, apresentando, portanto, a consistência do modelo. As variáveis que elevaram a confiabilidade do modelo, em caso de exclusão, foram as variáveis Governança de TI (GovTI), Portfólio de Projetos (PortProj) e a Formalização dos Projetos (FormProj).

\subsection{Análise de correlação}

Após verificar a consistência do modelo, foi realizada uma análise de correlação, que serve como pré-requisito para a análise de regressão e, consequentemente, verificação das hipóteses do estudo. A análise de correlação é apresentada na Tabela 3:

\section{Tabela 3}

Correlações

\begin{tabular}{l|r|r|r|r|r|r}
\hline \multicolumn{1}{c|}{ Variável } & \multicolumn{1}{c|}{ GovTI } & \multicolumn{1}{c|}{ PortProj } & IndicProj & ReavProj & EGP & FormProj \\
\hline GovTI & 1,000 & & & & & \\
PortProj & $0,559^{* * *}$ & 1,000 & & & &
\end{tabular}




\begin{tabular}{l|l|l|l|l|l|l} 
IndicProj & $0,563^{\star * *}$ & 0,539 & 1,000 & & & \\
ReavProj & $0,577^{\star * *}$ & 0,589 & 0,783 & 1,000 & & \\
EGP & $0,490^{\star * *}$ & 0,474 & 0,415 & 0,439 & 1,000 & 1,000 \\
\hline FormProj & $0,556^{\star * *}$ & 0,558 & 0,518 & 0,583 & 0,585 & 1,53 \\
\hline
\end{tabular}

Obs.: ${ }^{* * *} p \leq 0,01$.

Fonte: Dados da pesquisa, 2016.

Ao analisar as correlações, verifica-se que as práticas de gerenciamento de projetos estão correlacionadas com o índice de governança de $\mathrm{TI}$, ao nível de significância de $1 \%$. Isso sugere que todas as variáveis são importantes para compor o modelo.

\subsection{Análise de regressão}

Diante do resultado obtido na correlação, são colocados adiante os resultados da análise de regressão (Tabela 4), tendo como variável dependente o índice de governança de $\mathrm{TI}$ das instituições públicas e como variáveis independentes as variáveis que representam as práticas de gerenciamento de projetos. Para isso, foram gerados seis modelos distintos de regressão; em cada um dos modelos os valores referentes a uma determinada variável foram retirados:

\section{Tabela 4}

Resultados dos modelos de regressão

\begin{tabular}{|c|c|c|c|c|c|c|}
\hline Variáveis & $\begin{array}{c}(1) \\
\text { GovTI }\end{array}$ & $\begin{array}{c}(2) \\
\text { GovTI }\end{array}$ & $\begin{array}{c}(3) \\
\text { GovTI }\end{array}$ & $\begin{array}{c}(4) \\
\text { GovTI }\end{array}$ & $\begin{array}{c}(5) \\
\text { GovTI }\end{array}$ & $\begin{array}{c}(6) \\
\text { GovTI }\end{array}$ \\
\hline PortProj & $\begin{array}{c}0,156^{\star * \star} \\
(0,034)\end{array}$ & 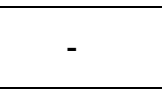 & $\begin{array}{c}0,169^{\star \star \star} \\
(0,035)\end{array}$ & $\begin{array}{c}0,173^{\star * *} \\
(0,033)\end{array}$ & $\begin{array}{c}0,176^{\star * *} \\
(0,035)\end{array}$ & $\begin{array}{c}0,183^{\star * \star} \\
(0,033)\end{array}$ \\
\hline IndicProj & $\begin{array}{c}0,155^{\star \star *} \\
(0,505)\end{array}$ & $\begin{array}{c}0,178^{* * *} \\
(0,051)\end{array}$ & - & $\begin{array}{c}0,225^{\star * *} \\
(0,039)\end{array}$ & $\begin{array}{c}0,165^{\star * *} \\
(0,052)\end{array}$ & $\begin{array}{c}0,163^{\star * *} \\
(0,050)\end{array}$ \\
\hline ReavProj & $\begin{array}{l}0,119^{\star \star} \\
(0,052)\end{array}$ & $\begin{array}{c}0,166^{* * *} \\
(0,051)\end{array}$ & $\begin{array}{c}0,227^{* * *} \\
(0,040)\end{array}$ & 2 & $\begin{array}{l}0,121^{* *} \\
(0,055)\end{array}$ & $\begin{array}{c}0,157^{\star * *} \\
(0,051)\end{array}$ \\
\hline EGP & $\begin{array}{c}0,137^{\star \star \star} \\
(0,029)\end{array}$ & $\begin{array}{c}0,175^{\star * *} \\
(0,028)\end{array}$ & $\begin{array}{c}0,106^{\star \star *} \\
(0,029)\end{array}$ & $\begin{array}{c}0,100^{\star * *} \\
(0,030)\end{array}$ & (5) & $\begin{array}{c}0,140^{\star \star \star} \\
(0,027)\end{array}$ \\
\hline FormProj & $\begin{array}{c}0,100^{\star \star \star} \\
(0,040)\end{array}$ & $\begin{array}{c}0,122^{\star * *} \\
(0,039)\end{array}$ & $\begin{array}{c}0,143^{\star * *} \\
(0,041)\end{array}$ & $\begin{array}{c}0,157^{\star \star \star} \\
(0,041)\end{array}$ & $\begin{array}{c}0,189^{\star * *} \\
(0,038)\end{array}$ & 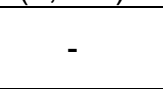 \\
\hline Constante $\left(\beta_{0}\right)$ & $\begin{array}{c}0,340 \\
(0,024)\end{array}$ & $\begin{array}{l}0,061^{* *} \\
(0,024)\end{array}$ & $\begin{array}{l}0,500^{* *} \\
(0,024)\end{array}$ & $\begin{array}{c}0,369 \\
(0,024)\end{array}$ & $\begin{array}{l}0,046^{*} \\
(0,024)\end{array}$ & $\begin{array}{l}0,042^{*} \\
(0,024)\end{array}$ \\
\hline $\mathrm{R}^{2}$ & 0,473 & 0,449 & 0,460 & 0,466 & 0,459 & 0,458 \\
\hline Estatística F & 62,05 & 68,34 & 72,01 & 77,59 & 75,24 & 73,10 \\
\hline Observações & 372 & 372 & 372 & 372 & 372 & 372 \\
\hline
\end{tabular}

Obs.: ${ }^{\star \star \star} p \leq 0,01 ;{ }^{* \star} p \leq 0,05 ;{ }^{*} p \leq 0,10$.

Fonte: Dados da pesquisa, 2016. 
Ao avaliar os dados dos modelos gerados, percebe-se que todas as variáveis relacionadas às práticas de gerenciamento de projetos foram relevantes para a melhoria da governança de $\mathrm{TI}$ das instituições públicas, mostrando-se assim significantes estatisticamente. Dentre as variáveis atribuídas, a variável Reavaliação de Projetos (ReavProj) é a que apresentou um menor índice de significância para o modelo, o que foi comprovado pela estatística $F$, que realiza a análise de variância, obtida em maior valor no modelo 4 da qual esta variável foi retirada. No modelo completo (1), esta variável apresentou significância estatística de $5 \%$, ante $1 \%$ das demais.

Em relação ao coeficiente de determinação $\left(R^{2}\right)$, foi verificado que, considerando o modelo em que todas as variáveis da pesquisa foram inseridas, obteve-se o valor de 0,473 . $O R^{2}$ se traduz na razão entre a variação explicada e a variação total dos dados, ou seja, ele procura explicar a fração da variação amostral em "y", que é explicada por " $x$ " (Wooldridge, 2010). No caso da pesquisa, observa-se que os $47,3 \%$ da variação do índice de governança de $\mathrm{TI}$ (GovTI) são explicados pelas variáveis relacionadas às práticas de gerenciamento de projetos inclusas no modelo. Nos demais modelos apresentados, não houve grandes variações nos resultados.

Para validar os resultados da regressão, foram realizados os testes que avaliam os pressupostos de ausência de multicolinearidade, homocedasticidade, variáveis omitidas e normalidade. Para verificar o pressuposto de ausência de multicolinearidade, foi realizado o teste do fator de inflação da variância (VIF), em que os valores próximos de 1 indicam baixos níveis de colinearidade (Hair Jr. et al., 2005). No modelo 1 , todas as variáveis independentes apresentaram valores baixos de VIF, com destaque para as variáveis PortProj e EGP, que apresentaram os valores 1,79 e 1,61, respectivamente.

Com relação à verificação da homocedasticidade dos resíduos, foi realizado o teste de Breusch-Pagan. A hipótese nula testada é de que os resíduos são homocedásticos, isto é, assume-se que a variância dos resíduos é constante (Wooldridge, 2010). Após realizar o teste, obteve-se um $p$-valor igual a 0,89 , o que rejeita a hipótese nula de homocedasticidade. Para corrigir o problema, foi considerado neste estudo o modelo de regressão robusto, válido para grandes amostras. 
No que se refere ao teste da existência de variáveis omitidas, foi realizado o teste de Ramsey, cuja hipótese nula é de que o modelo não possui variáveis omitidas. As variáveis omitidas são variáveis importantes para compor o modelo, mas que deixaram de ser incluídas. Assim, ao realizar o teste, obteve-se o $p$-valor de 0,67, não se podendo, assim, rejeitar a hipótese nula. Neste caso, é justificável a ausência de outras variáveis relacionadas às práticas de gerenciamento de projetos, visto que os dados utilizados na pesquisa são dados secundários, considerando a inviabilidade de incluir outras variáveis.

Por último, foi verificada a normalidade dos resíduos. A normalidade é importante, pois impacta a validade de todos os testes, incluindo as estatísticas t e f. Neste caso, assume-se que os resíduos têm distribuição normal e variância constante, sendo independentes das variáveis preditoras (Wooldridge, 2010). Para verificar este pressuposto, foi realizado o teste de Shapiro-Wilks, cuja hipótese nula é de que a distribuição dos resíduos é normal. O teste gerou um p-valor de 0,803, traduzindo em uma distribuição normal dos resíduos.

\subsection{Discussão dos resultados}

Com a validação dos pressupostos da regressão, bem como as variáveis que compõem o modelo, têm-se como resultado deste estudo que todas as hipóteses levantadas pelo modelo da pesquisa foram confirmadas pela análise de regressão. Este resultado pressupõe que as práticas de gerenciamento de projetos influenciam na melhoria do índice de governança de TI das instituições públicas analisadas pelo TCU. Vale destacar o gerenciamento de portfólio, a utilização de indicadores de desempenho dos projetos, a formalização das práticas de gerenciamento de projetos e a adoção de EGPs como variáveis que tiveram relevância estatística elevada nos modelos testados.

Em relação ao gerenciamento de portfólio, os resultados corroboram os estudos de Mosavi (2014) e Stentoft, Freytag e Thoms (2015). De fato, o gerenciamento de portfólio pode contribuir para a melhoria da governança, na medida em que as decisões, a comunicação e a negociação sobre a priorização de projetos envolvem membros da alta direção. No entanto, estas questões podem estar vinculadas à percepção de valor pela alta cúpula sobre os projetos e 
programas que devem ser priorizados, ou por fatores subjetivos, tais como influência política, levando, assim, à falta de padronização e coordenação destes mecanismos de gerenciamento (Stentoft et al., 2015). Portanto, é necessário entender esses aspectos, da mesma forma como são concebidas as estruturas de gerenciamento de portfólio e o grau de envolvimento da alta direção.

Sobre a questão de gerenciamento de indicadores, foi observado que uma destas questões se mostrou fortemente significante para o aumento da governança de TI das instituições, de acordo com outras pesquisas, como as de Bernardo (2014) e Liu e Wang (2016). Contudo, outra variável, relacionada ao gerenciamento de indicadores, que é a reavaliação dos projetos, embora tenha sido estatisticamente significante, não demonstrou uma contribuição forte para gerar uma melhor governança de TI destas instituições, o que pode indicar a falta de envolvimento ou estrutura de acompanhamento dos projetos pela alta direção, apesar de que esta questão ainda não possa ser comprovada.

Outras duas variáveis foram importantes para explicar o aumento da governança de TI: escritório de gerenciamento de projetos e formalização das práticas de gerenciamento de projetos, confirmando os resultados associados à literatura existente. Em relação aos escritórios, a literatura é mais enfática ao afirmar que essas estruturas fazem parte de uma estrutura maior de governança, na medida em que eles promovem maior controle dos projetos e funcionam como um instrumento de comunicação para as partes interessadas do projeto (Tsaturyan \& Muller, 2015; Aubry et al., 2014), embora seja mais comum o EGP realizar essas funções quando ele está localizado ou tem uma relação estreita com a alta direção, exercendo uma função mais estratégica e trabalhando, inclusive no gerenciamento de portfólio (Tsaturyan \& Muller, 2015; Blasevic et al., 2014).

Sobre a formalização do gerenciamento de projetos, os resultados da pesquisa vão ao encontro dos estudos existentes, embora ainda seja uma área pouco explorada, tendo o estudo de Muller e Martinsuo (2015) como um dos poucos que trabalham esta temática, mas associada a fatores de sucesso do projeto. De qualquer forma, é válido entender que mecanismos formais de acompanhamento, controle e alinhamento das práticas de gerenciamento de projetos com aspectos de governança tendem a melhorar o nível de sucesso os projetos (Muller \& Martinsuo, 2015). Essas questões precisam ser mais exploradas, no sentido de descobrir que 
mecanismos de formalização são mais utilizados para garantir a governança de TI destas instituições.

\section{Conclusões}

A pesquisa procurou avaliar em que medida a adoção de práticas de gerenciamento de projetos influencia no aumento da governança de TI das instituições públicas. Os resultados apontaram que todas as variáveis do modelo exercem influência sobre o aumento do índice de governança de TI das instituições analisadas, confirmando as hipóteses iniciais do estudo, sendo que as variáveis de gerenciamento de portfólio, a utilização de indicadores de desempenho dos projetos, a formalização das práticas de gerenciamento de projetos e a adoção de EGPs foram as que apresentaram maior significância estatística, confirmando, assim, as hipóteses da pesquisa.

Com base nesses resultados, é possível inferir que as instituições que investem mais em práticas de gerenciamento de projetos em TI conseguem obter maior controle desta área, considerando as questões relacionadas ao alinhamento estratégico, à transparência e à priorização de investimentos que gerem maior valor da TI do ponto de vista organizacional, sendo estas características importantes para medir a governança de $\mathrm{TI}$ das instituições referidas. Como implicações gerenciais para do presente estudo, ações podem ser desenvolvidas em relação ao uso mais intensivo destas práticas, no intuito de melhorar o uso dos recursos de $\mathrm{Tl}$ e sua contribuição para estas instituições.

Como limitações do estudo, destacam-se a ausência de variáveis que tratem de forma mais específica a situação atual destas práticas de gerenciamento de projetos, considerando o grau de explicação do modelo, comprovado por meio da análise do pressuposto de ausência de variáveis omitidas. Este problema não compromete a validade do estudo, porém, algumas variáveis importantes podem não ter sido avaliadas por não estarem contempladas na base de dados e no instrumento de avaliação proposto pelo TCU. Além disso, foi observado que a estrutura de escala adotada para avaliar as variáveis que compõem o perfil da governança de TI das instituições pelo TCU propicia um baixo nível de variação, mesmo que as questões tenham sido validadas, gerando, assim, a necessidade de 
reavaliação deste instrumento para obter um resultado mais aderente ao que se propõe investigar.

Como sugestões para pesquisas futuras, recomendam-se novos estudos quantitativos que analisem essa relação, levando em consideração aspectos específicos das práticas de gerenciamento de projetos e suas contribuições para a governança de TI, como, por exemplo, as estruturas de decisão e comunicação no gerenciamento de portfólio, conforme indicação da pesquisa de Mosavi (2014), ou nas funções relacionadas aos EGPs, explicitadas nas pesquisas de Aubry et al. (2007), Blasevic et al. (2014), e Patel et al. (2012). É importante levar em consideração também a necessidade de estudos qualitativos que demonstrem como estes indicadores influenciam na melhoria da governança de $\mathrm{TI}$, principalmente em aspectos até então pouco explorados, como a formalização das práticas de gerenciamento e os meios de acompanhamento e reavaliação dos projetos.

\section{Referências}

Ashraf, J., \& Uddin, S. (2015). New public management, cost savings and regressive effects: a case from a less developed country. Critical Perspectives on Accouting, 2015.

Aubry, M., Richer, M.-C., \& Lavoie-Tremblay, M. (2014). Governance performance in complex environment: the case of a major transformation in a university hospital. International Journal of Project Management, 32(8), 1333-1345.

Bekker, M. C. (2015). Project Governance - the definition and leadership dilemma. Social and Behavioral Sciences, 194 (2), 33-43.

Bernardo, M. R. (2014). Project indicators for enhancing governance of projects. Procedia Technology, 16, 1065-1071.

Bernroider, E. W. N., \& Ivanov, M. (2011). IT project management control and the Control Objectives for IT and related Technology (CobiT) framework. International Journal of Project Management, 29(3), 325-336.

Biesenthal, C., \& Wilden, R. (2014). Multi-level project governance: trends and opportunities. International Journal of Project Management, 32(8), 1291-1308.

Blasevic, G., Misic, S., \& Simac, M. (2014). Importance of managing PMO in Croatian PM market. Social and Behavioral Sciences, 119(19), 949-956. 
Brasil (2014). Relatório de Levantamento. Avaliação da Governança de Tecnologia da Informação na Administração Pública Federal. Subsídio às atividades de fiscalização do TCU. Relator: Ministro-Substituto Augusto Sherman Cavalcanti. Acórdão, 12 nov. 2014.

Burrel, G., \& Morgan, G. (1998). Sociological paradigms and organization analysis. Burlington: Ashgate Publishing.

Chang, C.-Y. (2015). Risk-bearing capacity as a new dimension to the analysis of project governance. International Journal of Project Management, 33(6), 11951205.

Corrar, L. J., Paulo, E., \& Dias Filho, J. M. (2007). Análise multivariada: para cursos de administração, ciências contábeis e economia. São Paulo: Atlas.

Dai, C. X., \& Wells, W. G. (2004). An exploration of project management office features and their relationship to project performance. International Journal of Project Management, 22(7), 523-532.

Diefenbach, T. (2007). The managerialistic ideology of organizational change management. Journal of Organizational Change Management, 20(1), 126-144.

Dinsmore, P. C. (2007). Como se tornar um profissional em gerenciamento de projetos: livro-base de preparação para certificação $\mathrm{PMP}^{\odot}$ - Project Management Professional (2a ed.). Rio de Janeiro: Qualitymark.

Guo, F. (2014). Effects of Project governance structures on the management of risks in major infrastructure projects: a comparative analysis. International Journal of Project Management, 32(5), 815-826.

Hair Jr, J. F., Anderson, R. E., Tatham, R. L., \& Black, W. C. (2005). Análise multivariada de dados. Porto Alegre: Bookman.

Heindrickson, G., \& Santos Jr., C. D. (2014). Information technology governance public organizations: how perceived effectiveness relates to three classical mechanisms. Journal of Information Systems and Technology Management, 11 (2), 297-326.

Hjelmbrekke, H., Laedre, O., \& Lohne, J. (2014). The need for a project governance body. International Journal of Managing Projects in Business, 7(4), 661-677.

Ho, J. L. Y., Wu, A., \& Xu, S. X. (2011). Corporate governance and returns on information technology investment: evidence from an emerging market. Strategic Management Journal, 32(6), 595-623.

Hobbs, B., \& Aubry, M. (2007). A multi-phase research program investigating project management offices (PMOs): the results of phase 1. Project Management Journal, 38(1), 74-86. 
Jalal, M. P., \& Koosha, S. M. (2015). Identifying organizational variables affecting project management office characteristics and analyzing their correlations in the Iranian project-oriented organizations of the construction industry. International Journal of Project Management, 33(2), 458-466.

Joslin, R., \& Muller, R. (2015). Relationships between a project management methodology and project success in different project governance contexts. International Journal of Project Management, 33, 6, 1377-1392.

Keil, M., Lee, H. K., \& Deng, T. (2013). Understanding the most critical skills for managing IT projects: a Delphi study of IT project managers. Information \& Management, 50(7), 398-414.

Levine, H. A. (2005). Project Portfolio Management: a pratical guide to selecting projects, managing portfolios, and maximizing benefits. San Francisco: JosseyBass.

Liu, S., \& Wang, L. (2016). Influence of managerial control on performance in medical information system projects: The moderating role of organizational environment and team risks. International Journal of Project Management, 34(1), 102-116.

Marconi, M. A., \& Lakatos, E. M. (2008). Fundamentos de Metodologia Científica. (6a ed.). São Paulo: Atlas.

Marnewick, C., \& Labuschagne, L. (2011). An investigation into the governance of information technology in South Africa. International Journal of Project Management, 29(6), 661-670.

Mendonça, C. M. C., Guerra, L. C. B., Sousa Neto, M. V., \& Araújo, A. G. (2013). Governança de tecnologia da informação: um estudo do processo decisório em organizações públicas e privadas. Revista de Administração Pública, 47(2), 443468.

Meredith, J. R., \& Mantel Jr., S. J. (2000). Project management: a managerial approach (4a ed.). New York: John Willey \& Sons.

Mohamed, N., \& Singh, J. K. A/P. (2012). A conceptual framework for information technology governance effectiveness in private organizations. Information Management \& Computer Security, 20(2), 88-106.

Mosavi, A. (2014). Exploring the roles of portfolio steering commitees in project portfolio governance. International Journal of Project Management, 32(3), 388399.

Muller, R., \& Lecoeuvre, L. (2014). Operationalizing governance categories of projects. International Journal of Project Management, 32(8), 1346-1357. 
Muller, R., \& Martinsuo, M. (2015). The impact of relational norms on information technology project success and its moderation through project governance. International Journal of managing Project in Business, 8(1), 154-176.

Pablo, A. L., Reay, T., Dewald, J. R., \& Casebeer, A. L. (2007). Identifying, enabling and managing dynamic capabilities in the public sector. Journal of Management Studies, 44(5), 687-708.

Patel, A. R., Patel, D. M., \& Patel, D. S. (2012). Implementation plan of PMO (Project Management Office) over EPMO (Enterprise Project Management Office) for beneficiaries success in today's organizations. International Journal of Research in Management and Technology, 2(6), 540-549.

Pinto, J. K. (2014). Project management, governance, and the normalization of deviance. International Journal of Project Management, 32(3), 376-387.

Prasad, A.; Heales, J.; Green, P. (2010). A capabilities-based approach to obtaining a deeper understanding of information technology governance effectiveness: evidence from IT steering committees. International Journal of Accounting Information Systems, 11(3), 214-232.

Prestes, A. M.; Brodbeck, A. F. (2011). Desenvolvimento de um conjunto de processos de governança de tecnologia da informação para uma instituição hospitalar. Revista Eletrônica de Sistemas de Informação, 10(2), 23-42.

Rolstadas, A., Tommelein, I., Schiefloe, P. M., \& Ballard, G. (2014). Understanding project success through analysis of project management approach. International Journal of managing Projects in Business, 7(4), 638-660.

Rosacker, K. M., \& Rosacker, R. E. (2010). Information technology project management within public sector organizations. Journal of Enterprise Information Management, 23(5), 587-594.

Rossetti, J. P. \& Andrade, A. (2011). Governança Corporativa: fundamentos, desenvolvimento e tendências (6a ed.). São Paulo: Atlas.

Secretaria de Fiscalização de Tecnologia da Informação (SEFTI). Fiscalização de tecnologia da informação. Recuperado de http://portal.tcu.gov.br/comunidades/fiscalizacao-de-tecnologia-dainformacao/atuacao/perfil-de-governanca-de-ti/.

Spalek, S. (2013). Improving industrial engineering performance through a successful project management office. Inzinerine Ekonomika-Engineering Economics, 24(2), 88-98.

Stentoft, J., Freytag, P. V., \& Thoms, L. (2015). Portfolio management of development projects in Danish municipalities. International Journal of Public Sector Management, 28(1), 11-28. 
Too, E. G., \& Weaver, P. (2014). The management of project management: a conceptual framework for project governance. International Journal of Project Management, 32(8), 382-1394.

Tsaturyan, T., \& Muller, R. (2015). Integration and governance of multiple project management offices (PMOs) at large organizations. International Journal of Project Management, 33(5), 1098-1110.

Turner, J. R. (2006). Towards a theory of project management: the nature of the project governance and project management. International Journal of Project Management, 24(2), 93-95.

Wooldridge, J. M. (2010). Introdução à econometria: uma abordagem moderna. São Paulo: Cengage Learning.

Veras, M. (2014). Gerenciamento de projetos: Project Model Canvas (PMC). Rio de Janeiro: Brasport.

Vergara, S. C. (2004). Projetos e relatórios de pesquisa em Administração (5a ed.). São Paulo: Atlas. 